\title{
RELATIONS AMONG CHARACTERISTIC CLASSES OF $n$-MANIFOLDS IMBEDDED IN $R^{n+k}$
}

\author{
STAVROS PAPASTAVRIDIS
}

\begin{abstract}
Let $I_{n} \subseteq H^{*}\left(B O ; Z_{2}\right)$ be the (graded) set of those normal characteristic classes which are zero on all compact, closed $C^{\infty}$ manifolds. Let $I_{n, k} \subseteq$ $H^{*}\left(B O ; Z_{2}\right)$ be the set of those characteristic classes which are zero on all $n$-manifolds which imbed in $R^{n+k}$. Let $K$ be the (graded) ideal in $H^{*}\left(B O ; Z_{2}\right)$ generated by the Stiefel-Whitney classes $w_{k}, w_{k+1}, w_{k+2}, w_{k+3}, \ldots$ We will prove the following result: If $1<i<\min \{(2 k-2),(n+k-1) / 2\}$, then $I_{n, k}^{i}=I_{n}^{i}+$ $K^{i}$. Also, we will prove an analogous result for manifolds with an extra structure.
\end{abstract}

Introduction. Let $f_{r}: X_{r} \rightarrow B O_{r}$ be a sequence of fibrations, with maps $g_{r}$ : $X_{r} \rightarrow X_{r+1}$, so that the usual diagram commutes. For such a situation $\mathrm{R}$. Lashof (see [2]) defines the concepts of $X$-structure of a manifold, $X$-characteristic class, $X$-cobordism and proves a Thom type theorem for cobordism classes of manifolds with an $X$-structure. From now on an " $X$-manifold" will be a closed compact $C^{\infty}$ manifold with an $X$-structure on its stable normal bundle. We call " $k-X$ manifold", an $n$-dimensional submanifold of $R^{n+k}$ with an $X$-structure on the normal bundle of the imbedding.

From now on $p$ will be a fixed prime number and all cohomology groups will be assumed with $Z_{p}$ coefficients. The set of mod-p (universal) $X$-characteristic classes is defined as $H^{*}(X)=$ proj $\lim H^{*}\left(X_{r}\right)$. Let $\gamma_{r}$ be the pull-back over $X_{r}$ of the universal $r$-dimensional vector bundle. We assume that the bundle $\gamma_{r}$ is $Z_{p}$-orientable for all $r$. Further we assume that, whenever $N$ is very big in comparison with $r$, then the obvious map $H^{*}\left(X_{N}\right) \rightarrow H^{*}\left(X_{r}\right)$ is onto in all dimensions and it is an isomorphism up to dimension $r$. Let $e_{k} \in H^{k}(X)$ be the unique element which goes to the Euler class (mod-p of course) of $\gamma_{k}$ by the obvious map $1_{k}: H^{k}(X) \rightarrow$ $H^{k}\left(X_{k}\right)$. (Of course the Euler class depends upon the selected $Z_{p}$-orientation of $\gamma_{k}$. But our result is independent of this choice.) From now on, $N$ will be a natural number which is taken very big compared to all other parameters and we put $X=X_{N}$ through the range being considered.

Definition 1. Let $I_{n} \subseteq H^{*}(X)$ be the set (graded) of those $X$-characteristic classes which go to zero by the normal map of all $n$-dimensional $X$-manifolds. (By "normal map" of an $X$-manifold $M$, we mean a lifting $M \rightarrow X_{N}$ of the Gauss map $M \rightarrow B O_{N}$, which is compatible with the $X$-structure of $M$.) Let $I_{n, k} \subseteq H^{*}(X)$ be the set (graded) of those $X$-characteristic classes which go to zero by the normal

Received by the editors November 9, 1978 and, in revised form, August 1, 1979.

AMS (MOS) subject classifications (1970). Primary 57R40, 57R20; Secondary 57R90, 55S10. 
map of all $n$-dimensional $k-X$-manifolds. (By "normal map of a $k-X$ manifold" $M$, we mean the composition $M \rightarrow X_{k} \rightarrow X_{N}$, where the first map is lifting of the Gauss map $M \rightarrow B O_{k}$ compatible with the $X$-structure of the normal bundle of the imbedded manifold $M$, and the second map is the obvious one.) The reader should take into consideration that in the definition above we identified the sets $H^{*}(X)$ and $H^{*}\left(X_{N}\right)$, which does no harm.

Finally, we assume that all spaces which appear in this paper are $\mathrm{CW}$-complexes, having a finite number of cells on each dimension.

Our main result is the following:

TheOREM 2. If $1<i<\min \{(2 k-2),(n+k-1) / 2\}$, then

$$
I_{n, k}^{i}=I_{n}^{i}+\left(\operatorname{ker} 1_{k}\right)^{i}+\left[e_{k}\right]^{i}
$$

([ $\left.e_{k}\right]$ is the (graded) ideal in $H^{*}(X)$ generated by $e_{k}$.)

The result stated in the abstract is the case $X_{r}=B O_{r}$. Theorem 2 includes also the cases $X_{r}=B S O_{r}, B U([r / 2]), B S U([r / 2]), B$ Spin, etc. For the cases $X_{r}=$ $\mathrm{BO}_{r}, \mathrm{BSO}_{r}, \mathrm{BU}([\mathrm{r} / 2]), \mathrm{I}_{n}$ has been computed by Brown and Peterson (see [1], and Brown-Peterson, Relations among characteristic classes. II, Ann. of Math. (2) 81 (1965), 356-363), so for those cases $I_{n, k}^{i}$ is completely known in the indicated range of dimensions.

Computations and proofs. In this section we will prove Theorem 2. We will split the proof in several preparatory lemmas, but first of all we need some terminology.

Obviously, we are losing nothing by assuming $i<n$, because the higher dimensional characteristic classes vanish on all $n$-manifolds. Besides we can assume that $2<k$ because for $k=1$ our theorem has no consequence.

From now on $N$ will be a positive integer which is very big in comparison with $n$ and $k$, so it does no harm to identify $H^{i}\left(X_{N}\right)$ and $H^{i}(X)$ for $i<N$, and with this understanding we call $1_{k}: H^{*}\left(X_{N}\right) \rightarrow H^{*}\left(X_{k}\right)$ the obvious projection. With this identification in mind, we have $e_{k} \in H^{k}\left(X_{N}\right)$. Further we can select Thom classes $U_{N} \in H^{N}\left(T \gamma_{N}\right)$ and $U_{k} \in H^{k}\left(T \gamma_{k}\right)$, so that $U_{k}$ reduces to $1_{k}\left(e_{k}\right)$, and $U_{N}$ goes to $\Sigma^{N-k} U_{k}$ under the obvious bundle map $\gamma_{k}+\varepsilon^{N-k} \rightarrow \gamma_{N}\left(\varepsilon^{N-k}\right.$ is the trivial $(N-k)$-dimensional bundle over $\left.X_{k}\right)$.

If $G$ is any group, then its dual is defined by $G^{*}=\operatorname{Hom}\left(G, Z_{p}\right)$. Let $K$ be Eilenberg-Mac Lane space $K\left(Z_{p}, n-i\right)$ (for $i=n$, then $K=$ point). Further we put $A=T \gamma_{k} \wedge K, A^{\prime}=T \gamma_{k} \wedge K_{+}, B=T \gamma_{N} \wedge K$ and $B^{\prime}=T \gamma_{N} \wedge K_{+} \cdot$ (Note that $K_{+}=K \cup$ (point $\}$.)

The first step, in order to prove Theorem 2, is to express in homotopy terms the geometrically defined sets $I_{n}$ and $I_{n, k}$. Following Brown-Peterson [1], we define the map $g: H^{i}\left(X_{n}\right) \rightarrow \pi_{N+n}^{*}(B)$, as follows: Let $a: S^{N+n} \rightarrow B$ be a map. Then for $x \in H^{i}\left(X_{N}\right)$ we define $g(x)$ by the formula $g(x)([a])=a^{*}\left(x U_{N} \wedge c\right)\left(\left[S^{N+n}\right]\right) \in$ $Z_{p}$, where $c$ is the fundamental class of $K$. Let $j_{1}: \pi_{N+n}^{*}(B) \rightarrow \pi_{N+n}^{*}\left(B^{\prime}\right)$ be the dual of the obvious map, let $j_{2}: \pi_{N+n}^{*}(B) \rightarrow \pi_{N+n}^{*}\left(\Sigma^{N-k} A\right)$ be the dual of the map induced by the bundle map $\gamma_{k}+\varepsilon^{N-k} \rightarrow \gamma_{N}$, let $\Sigma: \pi_{N+n}^{*}\left(\Sigma^{N-k} A\right) \rightarrow \pi_{n+k}^{*}(A)$ be 
the dual of the suspension, and finally let $j_{3}: \pi_{n+k}^{*}(A) \rightarrow \pi_{n+k}^{*}\left(A^{\prime}\right)$ be the dual of the obvious map.

LEMMA 3. $I_{n}^{i}=\operatorname{ker}\left(j_{1} g\right)$ and $I_{n, k}^{i}=\operatorname{ker}\left(j_{3} \Sigma j_{2} g\right)$.

Proof. The first assertion is Lemma (2.2) of [1]; the second one is proved analogously (see also [4]).

Next we will study the various maps which appear in Lemma 3.

LEMMA 4. If $i<(n+k-1) / 2$ then the map $\Sigma: \pi_{N+n}^{*}\left(\Sigma^{N-k} A\right) \rightarrow \pi_{n+k}^{*}(A)$ is a monomorphism. (Note that $A$ depends upon $i$.)

Proof. The reason is that the suspension map $\pi_{n+k}(A) \rightarrow \pi_{N+n}\left(\Sigma^{N-k} A\right)$ is onto in this range of dimensions (see [3, p. 458, Theorem 11]).

LEMMA 5. Let $A \stackrel{f}{\rightarrow} B \stackrel{g}{\rightarrow} C$ be an exact sequence of abelian groups, so that $C$ is finite and the elements of its $p$-component have order $p$. Then the dual sequence $C^{*} \rightarrow B^{*} \rightarrow A^{*}$ is also exact.

Proof. Let $t: B \rightarrow Z_{p}$ be a map so that $t f=0$. We will prove that there exists a map $T: C \rightarrow Z_{p}$ so that $T g=t$. Let $C_{p}$ be the subgroup of $C$ which consists of all elements of order $p$ and let $C_{0}$ be the subgroup of $C$ which consists of all elements of order prime to $p$. Then $C=C_{0} \oplus C_{p}$. Let $D_{p}=\operatorname{im}(g) \cap C_{p}$ and $D_{0}=\operatorname{im}(g) \cap$ $C_{0}$; then $\operatorname{im}(g)=D_{0} \oplus D_{p}$. But since $C_{p}$ is a $Z_{p}$-space, then $D_{p}$ splits, i.e. there is a subgroup $E$ of $C_{p}$, such that $C_{p}=D_{p} \oplus E$. Finally we define the map $T$, in the following manner: If $x \in C_{0}$ or $E$ then $T(x)=0$. If $x \in D_{p}$ and $x=g(y)$, then $T(x)=t(y)$. It can be proved without difficulty that $T$ is well defined and that $T g=t$.

LEMMA 6. Let $Z$ be an $(r-1)$-connected space, such that $\bar{H}^{*}(Z)$ is a free $A_{p}$-module up to dimension $s+1$ and $\max (s, 2)<2 r$. (Note that $A_{p}$ is the mod-p Steenrod algebra.) Then the following things occur:

(i) If $i<s$, then $\pi_{i}(Z)$ is finite and all the elements of its p-component have order $p$.

(ii) If $i<s$, then the dual of the mod-p Hurewicz homomorphism $H^{i}(Z) \rightarrow \pi_{i}^{*}(Z)$ is onto.

(iii) Let $x_{1}, x_{2}, x_{3}, \ldots, x_{m}$ be a set of elements which is an $A_{p}$-basis of $\bar{H}^{*}(Z)$ up to dimension s. We assume that $i<s$. Then for every $f \in \pi_{i}^{*}(Z)$, there is at least one $z \in H^{i}(Z)$ which is a $Z_{p}$-linear combination of $x_{1}, x_{2}, \ldots, x_{m}$, such that if $a$ : $S^{i} \rightarrow Z$, then $f([a])=a^{*}(z)\left(\left[S^{i}\right]\right)$.

Proof. By the assumptions, there is a map $Z \rightarrow$ product of $K\left(Z_{p^{\prime}}\right)$ 's which induces an isomorphism in $Z_{p}$-cohomology up to dimension $s+1$. From this all the rest follows without difficulty.

LEMMA 7. Let $X \rightarrow Z \rightarrow Z / X$ be a cofibration of simply-connected spaces such that the following conditions are satisfied:

(a) The map $H^{*}(Z) \rightarrow H^{*}(X)$ is onto in all dimensions and it is mono up to dimension $(r-1)$. 
(b) The map $\pi_{*}(Z, X) \rightarrow \pi_{*}(Z / X, *)$ is an isomorphism up to dimension $s$ where $s<2 r$.

(c) The kernel of the map $H^{*}(Z) \rightarrow H^{*}(X)$ is a free $A_{p}$-module up to dimension $s+1$. Let $x_{1}, x_{2}, \ldots, x_{m}$ be an $A_{p}$-basis of this module.

Then, if $f$ is an element of the kernel of the map $\pi_{i}^{*}(Z) \rightarrow \pi_{i}^{*}(X)$ and $i<s$, then there is a $Z_{p}$-linear combination of $x_{1}, \ldots, x_{m}$, let it be $z \in H^{i}(Z)$, such that if $a$ : $S^{i} \rightarrow Z$ then $f([a])=a^{*}(z)\left(\left[S^{i}\right]\right)$.

Proof. By assumption, if $i \leqslant s$ then we have an exact sequence $\pi_{i}(X) \rightarrow \pi_{i}(Z) \rightarrow$ $\pi_{i}(Z / X)$. Besides, $\bar{H}^{*}(Z / X)=\operatorname{ker}\left(H^{*}(Z) \rightarrow H^{*}(X)\right)$ satisfies the assumptions of the previous lemma, so by Lemma 5 we get an exact seqeunce $\pi_{i}^{*}(Z / X) \rightarrow \pi_{i}^{*}(Z)$ $\rightarrow \pi_{i}^{*}(X)$. Then our lemma follows by applying Lemma 6(iii).

Let $p_{1}: \pi_{n+k}\left(A \times T \gamma_{k}\right) \rightarrow \pi_{n+k}(A)$ be the obvious projection. Then we have the following

LEMMA 8. The dual of the projection $p_{1}^{*}: \pi_{n+k}^{*}(A) \rightarrow \pi_{n+k}^{*}\left(A \times T \gamma_{k}\right)$ is a monomorphism.

Proof. Obvious, because $p_{1}$ is onto.

Let $t$ be the obvious map $t: A^{\prime} \rightarrow A \times T \gamma_{k}$. Then we have the following lemma:

LEMMA 9. The map $t^{*}$ is onto in $Z_{p}$-cohomology and its kernel is generated by all the elements of the form

$$
x \wedge z \otimes y+(-1)^{\operatorname{deg} z \cdot \operatorname{deg} y} x y \wedge z \otimes 1,
$$

for all $x, y \in \bar{H}^{*}\left(T \gamma_{k}\right)$ and $z \in \bar{H}^{*}(K)$. Furthermore, we have

(i) The map $\pi_{n+k}\left(A \times T \gamma_{k}, A^{\prime}\right) \rightarrow \pi_{n+k}\left(\left(A \times T \gamma_{k}\right) / A^{\prime}\right.$, *) is an isomorphism if $i \leqslant 2 k-2$.

(ii) The kernel of the map $t^{*}: H^{*}\left(A \times T \gamma_{k}\right) \rightarrow H^{*}\left(A^{\prime}\right)$ is a free $A_{p}$-module up to dimension $2 k+2(n-i)$. Further, let $x_{1}, x_{2}, \ldots, x_{m}$ be a $Z_{p}$-basis of $\bar{H}^{*}\left(T \gamma_{k}\right)$. Then the set of elements

$$
x_{i} \wedge c \otimes x_{j}+(-1)^{\operatorname{deg} c \cdot \operatorname{deg} x_{j}} x_{i} x_{j} \wedge c \otimes 1
$$

is an $A_{p}$-basis of this kernel. ( $c$ is the fundamental class of $K$.)

(iii) Let $f$ be an element of the kernel of the map $t_{*}^{*}: \pi_{n+k}^{*}\left(A \times T \gamma_{k}\right) \rightarrow \pi_{n+k}^{*}\left(A^{\prime}\right)$ and let $i<\min \{(2 k-2),(n+k-1) / 2\}$. Then there is a $y \in\left[E_{k}\right]^{i}$ such that, for every $a: S^{n+k} \rightarrow A \times T \gamma_{k}$, we have

$$
f([a])=a^{*}\left(\left(y U_{k} \wedge c\right) \otimes 1\right)\left(\left[S^{n+k}\right]\right)
$$

( $E_{k}$ is the Euler class of $\gamma_{k}$ and $\left[E_{k}\right]$ is the ideal in $H^{*}\left(X_{k}\right)$ generated by $E_{k}$ ).

Proof. It is easy to determine the image and the kernel of $t^{*}$ by applying the Künneth formula. Besides part (i) comes from the fact that the couple $(A \times$ $\left.T \gamma_{k}, A^{\prime}\right)$ is $(2 k+(n-i)-1)$-connected and that $A^{\prime}$ is $(k-1)$-connected by applying homotopy excision (see [3, p. 487, 6 Corollary]). Part (ii) comes from the structure of $\operatorname{ker}\left(t^{*}\right)$ described above. Part (iii) follows from Lemma 7 and the fact that $x_{i} x_{j}=a_{i} U_{k} a_{j} U_{k}= \pm a_{i} a_{j} U_{k} U_{k}= \pm a_{i} a_{j} E_{k} U_{k}$, and that the element $x_{i} \wedge c \otimes$ $x_{j}$ is decomposable in $H^{*}\left(A \times T \gamma_{k}\right)$. 
Consider the natural map $\Sigma^{N-k} T \gamma_{k} \rightarrow T \gamma_{N}$ which comes from the Thomification of the bundle map $\gamma_{k}+\varepsilon^{N-k} \rightarrow \gamma_{N}$. This map is onto in $Z_{p}$-cohomology and its kernel is $\left(\operatorname{ker} 1_{k}\right) U_{N}$. This map induces in an obvious way the map $\Sigma^{N-k} T \gamma_{k} \wedge K$ $\rightarrow T \gamma_{N} \wedge K$, i.e. $\Sigma^{N-k} A \rightarrow B$, which is also onto in $Z_{p}$-cohomology and its kernel is (ker $\left.1_{k}\right) U_{N} \wedge \bar{H}^{*}(K)$. But this kernel is a free $A_{p}$-module up to dimension $2(n-i)$ $+k+1+N$ and there is an $A_{p}$-basis formed by elements of the form $x U_{N} \wedge c, x$ runs over a basis of ker $1_{k}$. From the remarks above, the following lemma follows easily.

LEMMA 10. Let $f$ be an element of the kernel of the map $\pi_{N+n}^{*}(B) \rightarrow \pi_{N+n}^{*}\left(\Sigma^{N-k} A\right)$. Then there is an element $z \in\left(\operatorname{ker}^{\prime} 1_{k}\right)^{i}$, so that for every $a: S^{N+n} \rightarrow B$ we have

$$
f([a])=a^{*}\left(z U_{N} \wedge c\right)\left(\left[S^{N+n}\right]\right) \text {. }
$$

And now with all this preparation, Theorem 2 follows without difficulty.

Proof OF THEOREM 2. Let us consider the following diagram

$$
\begin{aligned}
& \pi_{n+k}^{*}\left(A \times T \gamma_{k}\right) \stackrel{t^{*}}{\rightarrow} \quad \pi_{n+k}^{*}\left(A^{\prime}\right) \\
& p_{1}^{* \uparrow} \quad \nearrow_{j_{3}} \\
& \pi_{n+k}^{*}(A) \\
& \Sigma \uparrow \\
& \pi_{N+n}^{*}\left(\Sigma^{N-k} A\right) \\
& j_{2} \uparrow \\
& H^{i}\left(X_{N}\right) \stackrel{g}{\rightarrow} \quad \pi_{N+n}^{*}(B) \quad \stackrel{j_{1}}{\rightarrow} \quad \pi_{N+n}^{*}\left(B^{\prime}\right)
\end{aligned}
$$

Let $x$ be an element of $I_{n, k}^{i}$; then (by Lemma 3) $j_{3} \Sigma j_{2} g(x)=0$. But obviously $j_{3}=t_{*}^{*} p_{1}^{*}$, so $t_{*}^{*}\left[p_{1}^{*} \Sigma j_{2} g(x)\right]=0$. But (by Lemma 9(iii), and the definition of $g$ ) there is an element $y \in\left[e_{k}\right]^{i}$ so that $p_{1}^{*} \Sigma j_{2} g(x-y)=0$. But (by Lemmas 4 and 3) this implies that $j_{2} g(x-y)=0$. But (by Lemma 10) this means that there is an element $z$ of $\left(\operatorname{ker} 1_{k}\right)^{i}$ such that $g(x-y-z)=0$. But Lemma 3 implies that $(x-y-z) \in I_{n}^{i}$. That ends the proof.

\section{REFERENCES}

1. E. Brown and F. Peterson, Relations among characteristic classes. I, Topology 3 (1964), 39-52.

2. R. Lashof, Poincaré duality and cobordism, Trans. Amer. Math. Soc. 109 (1963), 257-277.

3. E. Spanier, Algebraic topology, McGraw-Hill, New York, 1966.

4. R. Thom, Quelques propriétés globales des variétés différentiables, Comment. Math. Helv. 28 (1954), 17-86.

Department of Mathematics, University of Patras, Patras, Greece 